\title{
Participation of the Third Order Optical Nonlinearities in Nanostructured Silver Doped Zinc Oxide Thin Solid Films
}

\author{
C. Torres-Torres, ${ }^{1}$ L. Castañeda, ${ }^{2}$ M. Trejo-Valdez, ${ }^{3}$ A. Maldonado, ${ }^{4}$ and R. Torres-Martínez ${ }^{5}$ \\ ${ }^{1}$ Sección de Estudios de Posgrado e Investigación, ESIME-Z, Instituto Politécnico Nacional, 07738 México, DF, Mexico \\ ${ }^{2}$ Escuela Superior de Ingeniería Mecánica y Eléctrica Unidad Ticomán, Instituto Politécnico Nacional, 07340 México, DF, Mexico \\ ${ }^{3}$ ESIQIE, Instituto Politécnico Nacional, 07738 México, DF, Mexico \\ ${ }^{4}$ Departamento de Ingeniería Eléctrica, CINVESTAV IPN-SEES, A. P. 14740, 07000 México, DF, Mexico \\ ${ }^{5}$ Centro de Investigación en Ciencia Aplicada y Tecnología Avanzada Unidad Querétaro, Instituto Politécnico Nacional, \\ 76090 Santiago de Querétaro, QRO, Mexico
}

Correspondence should be addressed to L. Castañeda, luiscas@cablevision.net.mx

Received 9 October 2012; Accepted 19 November 2012

Academic Editor: Masayuki Nogami

Copyright (C) 2012 C. Torres-Torres et al. This is an open access article distributed under the Creative Commons Attribution License, which permits unrestricted use, distribution, and reproduction in any medium, provided the original work is properly cited.

We report the transmittance modulation of optical signals in a nanocomposite integrated by two different silver doped zinc oxide thin solid films. An ultrasonic spray pyrolysis approach was employed for the preparation of the samples. Measurements of the third-order nonlinear optical response at a nonresonant $532 \mathrm{~nm}$ wavelength of excitation were performed using a vectorial twowave mixing. It seems that the separated contribution of the optical nonlinearity associated with each film noticeable differs in the resulting nonlinear effects with respect to the additive response exhibited by the bilayer system. An enhancement of the optical Kerr nonlinearity is predicted for prime number arrays of the studied nanoclusters in a two-wave interaction. We consider that the nanostructured morphology of the thin solid films originates a strong modification of the third-order optical phenomena exhibited by multilayer films based on zinc oxide.

\section{Introduction}

The attractive features related to nonlinear optical media are mainly given by the potential applications in diverse areas for optical communications, instrumentation techniques, sensors, actuators, and processing of signals, among other purposes [1]. Certainly, all materials can exhibit a third order optical nonlinearity; but those that are functionally considered third-order optical materials, in a higher level, are capable to present noteworthy changes in absorptive and refractive effects with dependence on irradiance excitations. Several scientific researches about distinct processing routes for preparing advanced optical materials have been carried out [2]; however, the powerful and ultrafast optical response of nanostructures has given rise to their relevance in the development of low-dimensional nonlinear optical devices for photonic effects or electrical phenomena [3]. In this respect, one of the most important physical behaviors responsible of the modification of the optical features of nanoparticles is given by the quantum confinement, which originates a discrete and sharp selective participation of the electronic nature in a sample with physical properties strongly dependent on size and shape [4]. Besides, it also has been demonstrated that a reinforcement of particular optical processes can be engineered by quantum conditions [5]. Apparently, the influence of the environment on the nanoparticles also should be taken into account in order to better describe their optical and electrical characteristics $[6,7]$. Therefore, with the inclusion of dopants to produce changes in the morphology of a nanostructured material, it is expected to accomplish the possibility to tailor the optical nonlinearities [8]. Zinc oxide $(\mathrm{ZnO})$ is a promising transparent material with fascinating optical and photoconductive properties $[9,10]$. An outstanding participation of silver $(\mathrm{Ag})$ ions in the improved optical response of $\mathrm{ZnO}$ thin films has been pointed out [11]. Thus, in this work, we 
analyze a nonlinear optical experiment with a nonresonant excitation in Ag doped $\mathrm{ZnO}$ samples. We study the nonlinear optical response of two different thin solid films in order to make notice of the resulting nonlinearity dependent on the duplication of the thickness of the film. Finally, we find that a prime number array of the studied nanoclusters in a twowave interaction can generate an important enhancement of the optical Kerr effect.

\section{Experimental Details}

2.1. Processing Route of the Samples. $\mathrm{ZnO}: \mathrm{Ag}$ thin solid films were deposited from a $0.4 \mathrm{M}$ solution of zinc (II) acetate $\left[\mathrm{Zn}\left(\mathrm{O}_{2} \mathrm{CCH}_{3}\right)_{2}\right]$ (from Alfa, 98\%), dissolved in a mix of deionized water, acetic acid $\left[\mathrm{CH}_{3} \mathrm{CO}_{2} \mathrm{H}\right]$ (from Baker, 98\%), and methanol $\left[\mathrm{CH}_{3} \mathrm{OH}\right]$ (from Baker, 98\%) (100: 100:800 volume proportion). Separately, a $0.2 \mathrm{M}$ solution of silver nitrate $\left[\mathrm{Ag}\left(\mathrm{NO}_{3}\right)\right]$ (from Baker, 98\%) dissolved in a mix of deionized water and acetic acid $\left[\mathrm{CH}_{3} \mathrm{CO}_{2} \mathrm{H}\right]$ (from Baker, $98 \%$ ) (1:1 vol. proportion) was prepared, in order to be used as doping source. A constant $[\mathrm{Ag}] /[\mathrm{Zn}]$ ratio of 2 at. \% was used. The deposition system used in this work includes a piezoelectric transducer operating at variable frequency, which was set to $1.2 \mathrm{MHz}$ and the ultrasonic power at $120 \mathrm{~W}$. $\mathrm{ZnO}: \mathrm{Ag}$ films were deposited onto sodalime glass substrates with an area of $2.0 \times 1.0 \mathrm{~cm}^{2}$. The details of the deposition system have been previously described [12]. The cleaning process of the substrates is as follows: (i) sonication for 5 minutes in trichloroethylene $\left(\left(\mathrm{C}_{2} \mathrm{HCl}_{3}\right)\right.$ from Baker, 98\%) for degreasing the substrates, followed by (ii) sonication in methyl alcohol $\left(\left(\mathrm{CH}_{3} \mathrm{OH}\right)\right.$ from Aldrich, $98 \%)$, (iii) sonication in acetone $\left(\left(\mathrm{CH}_{3} \mathrm{COCH}_{3}\right)\right.$ from Baker, $98 \%$ ), and finally, (iv) the substrates are dried by a jet of pure and dry nitrogen $\left(\left(\mathrm{N}_{2}\right)\right.$ from PRAXAIR, 99.997\%). Then the substrates were placed on a fused tin bath, whose temperature was measured just below the substrate using a chromel-alumel thermocouple, which is contained in a stainless steel metal jacket. The substrate temperature $\left(T_{s}\right)$ was $400^{\circ} \mathrm{C}$, within an accuracy of $\pm 0.5^{\circ} \mathrm{C}$. Pure $\mathrm{N}_{2}$ (from PRAXAIR, 99.997\%) was used as the solution carrier and director gas, with flow rates of 3.5 and $0.5 \mathrm{~L} / \mathrm{min}$, respectively. In order to promote the crystallization, the samples were annealed at $400^{\circ} \mathrm{C}$ in a $\mathrm{N}_{2}$ atmosphere showing a better densification traduced as a reduction of particle size when the annealing lasts 4 hrs. The thickness of the deposited films onto soda-lime glass was manipulated by controlling the time of deposition. The film thickness obtained after 10 and 5 minutes of deposition was measured with a KLA Tencor P15 profilometer, resulting in approximately 500 $\mathrm{nm}$ and $250 \mathrm{~nm}$, respectively. The morphology of the thin films was analyzed using Scanning Electronic Microscopy (SEM) performed with a FEI Quanta 3D FEG Microscope system in STEM mode (Scanning Transmission Electronic Microscopy). The elemental composition of the samples was obtained by Auger Electron Spectroscopy (AES) using a JAMP-7800 (JEOL) equipment, with a base pressure of $8.6 \times$ $10^{-8} \mathrm{~Pa}\left(6.15 \times 10^{-10}\right.$ torr $)$. The parameters of the primary electron beam were $10 \mathrm{keV}$ of energy and $0.34 \mu \mathrm{A}$ of current. The samples were inclined at $55^{\circ}$ with respect to the normal

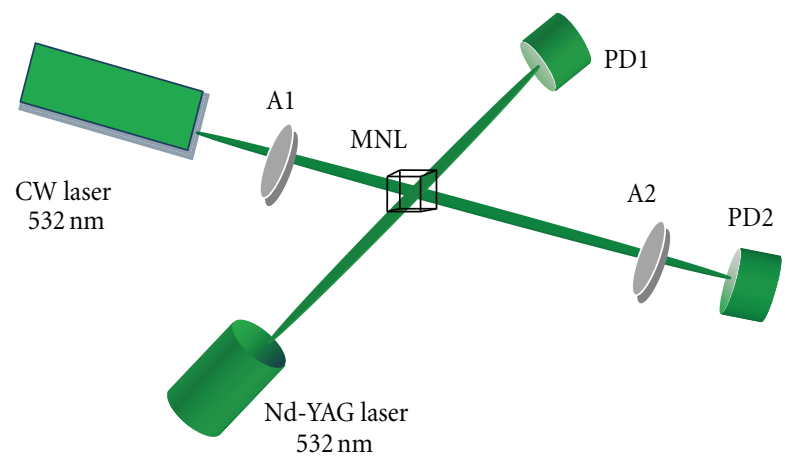

FIGURE 1: Schematic illustration of the experimental setup for the vectorial two-wave interaction.

of the surface. The linear transmittance spectra of the thin films were acquired with a Perkin Elmer XLS UV-visible spectrophotometer.

2.2. Third Order Nonlinear Optical Response. The annealed thin solid film samples were investigated measuring the optical transmittance in a vectorial two-wave mixing experiment. Separated measurements on different thin solid films with thickness of $500 \mathrm{~nm}$ and $250 \mathrm{~nm}$ were performed. Then, we measured a tandem configuration integrated initially by two films with $250 \mathrm{~nm}$ thickness put together to obtain a $500 \mathrm{~nm}$ sample width; finally another tandem configuration was measured in the same fashion with the two films of $500 \mathrm{~nm}$ thickness giving a $1000 \mathrm{~nm}$ thickness of total sample width. Figure 1 illustrates the experimental setup. MNL represents the sample, A1-2 are polarizers, and PD1-2 are photodetectors with integrated filters. Pump and probe beams, with linear polarizations making an angle of $45^{\circ}$, are in simultaneous propagation in the same region of interaction of the studied sample. A solid state laser with $1.5 \mathrm{~W}$ of average power and $532 \mathrm{~nm}$ wavelength was employed as a probe beam; whereas a nanosecond Nd:YAG laser with $532 \mathrm{~nm}$ wavelength and maximum energy of $375 \mathrm{~mJ}$ per pulse at $1 \mathrm{~Hz}$ was employed as a pump. The diameter of the beam waist for the probe beam in the sample was measured to be $1 \mathrm{~mm}$ and for the pump beam was $6 \mathrm{~mm}$.

In order to describe the propagation of the electric fields in the two-wave interaction taking into account a null selfdiffraction phenomenon, we follow the procedure to obtain the expressions for the amplitudes of the transmitted fields derived elsewhere [13]

$$
\begin{aligned}
& E_{1 \pm}(z)=\left[E_{1 \pm}^{0} J_{0}\left(\Psi_{ \pm}^{(1)}\right)+i E_{2 \pm}^{0} J_{1}\left(\Psi_{ \pm}^{(1)}\right)\right] \exp \left(-i \Psi_{ \pm}^{(0)}-\frac{\alpha z}{2}\right) \\
& E_{2 \pm}(z)=\left[E_{2 \pm}^{0} J_{0}\left(\Psi_{ \pm}^{(1)}\right)-i E_{1 \pm}^{0} J_{1}\left(\Psi_{ \pm}^{(1)}\right)\right] \exp \left(-i \Psi_{ \pm}^{(0)}-\frac{\alpha z}{2}\right)
\end{aligned}
$$

where $E_{1 \pm}(z)$ and $E_{2 \pm}(z)$ are the complex amplitudes of the circular components of the transmitted waves beams; while $E_{1 \pm}^{0}$ and $E_{2 \pm}^{0}$ are the amplitudes of the incident waves at the surface of the sample; $\alpha$ is the optical absorption coefficient; 


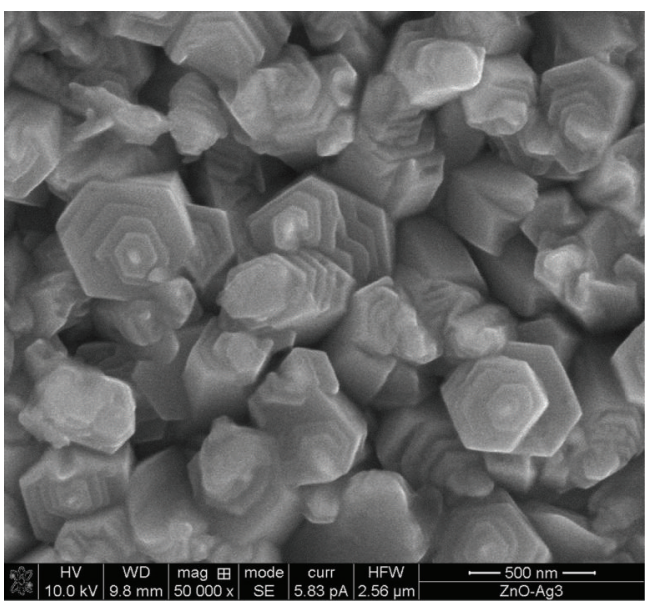

(a)

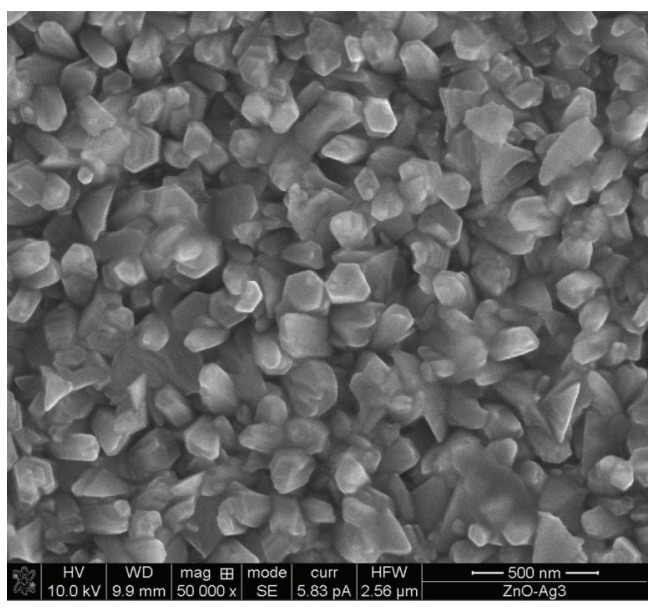

(b)

Figure 2: Typical SEM micrograph of the $\mathrm{ZnO}$ : Ag films (a) as-deposited (b) after annealing at $400^{\circ} \mathrm{C}$ in nitrogen.

$J_{m}\left(\Psi_{ \pm}^{(1)}\right)$ stands for the Bessel function of order $m, z$ is the thickness of the nonlinear media, and

$$
\begin{gathered}
\Psi_{ \pm}^{(0)}=\frac{4 \pi^{2} z}{n_{0} \lambda}\left[A \sum_{j=1}^{2}\left|E_{j \pm}\right|^{2}+(A+B) \sum_{j=1}^{2}\left|E_{j \mp}\right|^{2}\right], \\
\Psi_{ \pm}^{(1)}=\frac{4 \pi^{2} z}{n_{0} \lambda}\left[A E_{1 \pm} E_{2 \pm}^{*}+(A+B) E_{1 \mp} E_{2 \mp}^{*}\right]
\end{gathered}
$$

are the nonlinear phase changes. Here $A=6 \chi_{1122}^{(3)}$ and $B=$ $6 \chi_{1221}^{(3)}$, which are the independent components of the thirdorder susceptibility tensor $\chi^{(3)}$ for an isotropic material. The optical wavelength is represented by $\lambda$ and the refractive index is $n_{o}$.

\section{Results and Discussion}

From Figure 2, it can be observed a representative panoramic view of one region of the samples obtained by Scanning Electronic Microscopy (SEM). Both images show evidence of the nanostructured morphology that can be obtained with the ultrasonic spray pyrolysis deposition technique. The grain size distribution range results in grains with $400 \mathrm{~nm}$ to $500 \mathrm{~nm}$ for the as-deposited sample, while these grain size distributions are reduced by the annealing process as it has been previously indicated [12] resulting in values from $100 \mathrm{~nm}$ to $200 \mathrm{~nm}$. The surface morphology of $\mathrm{ZnO}$ : Ag thin films is composed of individual grains resembling slices with hexagonal shape; it can also be noted that secondary grains are formed by stacks of slices, on top of which there is a kind of spiral. This type of defects can be identified as screw dislocations. It is usual to find out similar characteristics in $\mathrm{ZnO}$ thin films deposited by ultrasonic spray pyrolysis [14]. Columnar grains with a hexagonal shaped form were observed in both cases.

The doping concentration through the films was determined by AES. A uniform distribution of the Ag and an absence of segregation were observed. The Ag content within

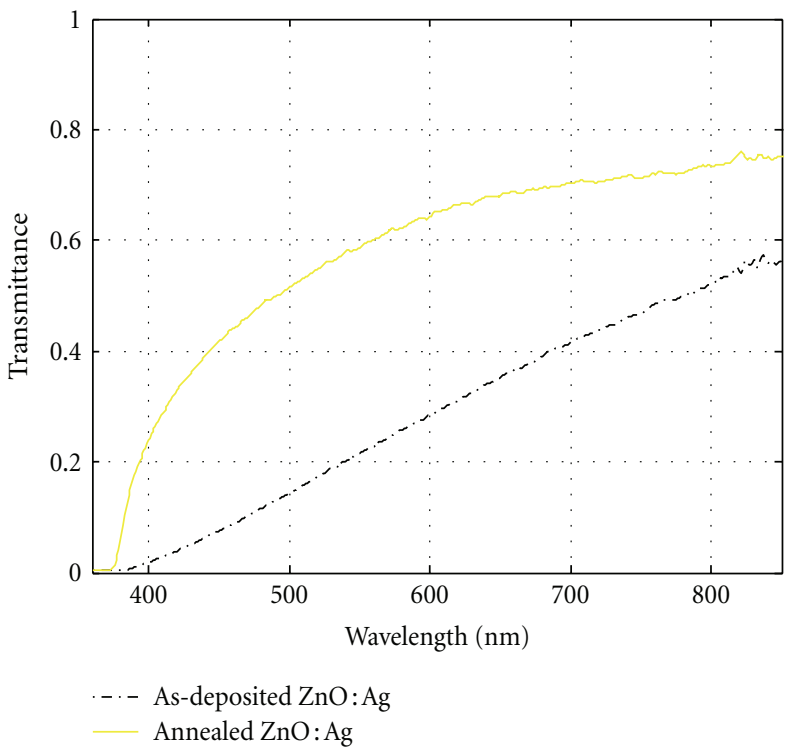

Figure 3: Transmittance spectra of the samples.

the films was always below the values corresponding to the starting solution, possibly due to the evaporation of an $\mathrm{Ag}$ fraction during the film growth. However, it was confirmed that the annealing process does not participate in the evaporation of Ag after the deposition process. The resulting distribution of $\mathrm{Ag}$ in the $\mathrm{ZnO}$ : $\mathrm{Ag}$ samples were found to be close 1.0 at.\%. Finally a stoichiometric relationship between $\mathrm{Zn}$ and $\mathrm{O}$, corresponding to $\mathrm{ZnO}$ was observed throughout the whole films.

The corresponding linear transmittance spectra obtained for the studied $\mathrm{ZnO}: \mathrm{Ag}$ thin solid films are shown in Figure 3. One can clearly notice a significant effect of transparency resulting from the annealing process in the film for wavelengths above about $400 \mathrm{~nm}$ in the plot.

The polarized irradiances of the transmitted beams in the two-wave interaction were measured in different cases 


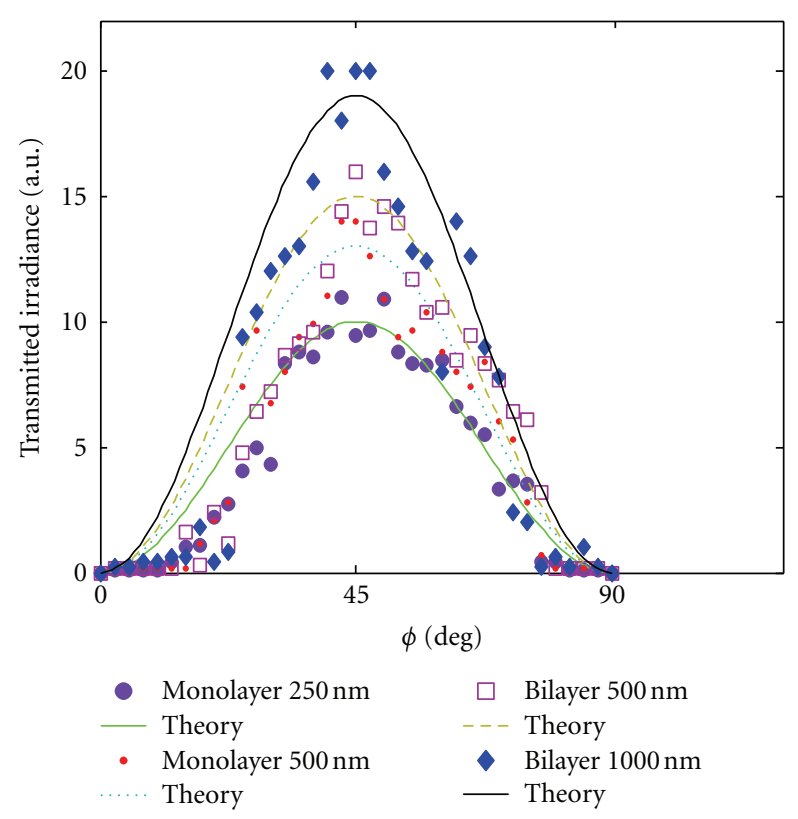

FIgURE 4: Nonlinear optical results.

TABLE 1: Magnitude of the third-order optical susceptibility in the studied samples.

\begin{tabular}{lccc}
\hline & \multicolumn{2}{c}{$\left|\chi^{(3)}\right|[\mathrm{esu}]$} \\
Monolayer & Monolayer & Bilayer & Bilayer \\
$250 \mathrm{~nm}$ & $500 \mathrm{~nm}$ & $500 \mathrm{~nm}$ & $1000 \mathrm{~nm}$ \\
\hline $4.4 \times 10^{-9}$ & $1.9 \times 10^{-9}$ & $3.7 \times 10^{-9}$ & $1.8 \times 10^{-9}$ \\
\hline
\end{tabular}

of polarization of the incident beams making an angle $\varphi$ between their planes of polarization. The axes of transmission of the analyzers A1-2 were aligned in order to detect the orthogonal components of the polarization of the probe waves. An error bar of $\pm 10 \%$ was estimated for the experimental irradiance data. By comparing numerical simulations of (1)-(4) with the data obtained from the transmitted irradiances in the thin film samples, we obtained the nonlinear optical parameters. Figure 4 shows the experimental transmittance of the probe beam with the best fitting of the numerical simulations.

The estimated $\left|\chi^{(3)}\right|$ parameters are presented in Table 1.

As a comparative result, we measured the nonlinear optical response for a pure $\mathrm{ZnO}$ thin solid film sample and then we find that $\left|\chi^{(3)}\right|=6.2 \times 10^{-11}$ esu. These last results are comparable with the nanosecond nonlinear optical response previously reported for $\mathrm{ZnO}$ and $\mathrm{ZnO}$ : $\mathrm{Ag}$ samples were prepared with different processing techniques [15].

Numerical simulations made by (1)-(4) allow us to compare the nonlinear optical enhancement related to the spatial period of the distributions of the nanoclusters in a bilayer $\mathrm{ZnO}$ thin solid film. We consider that the first and the second layer display periodic periods, $T_{A}$ and $T_{B}$, respectively. Figure 5 illustrates the variation in the predicted nonlinear optical response for the studied samples when we consider that a periodic distribution of nanoclusters can be founded

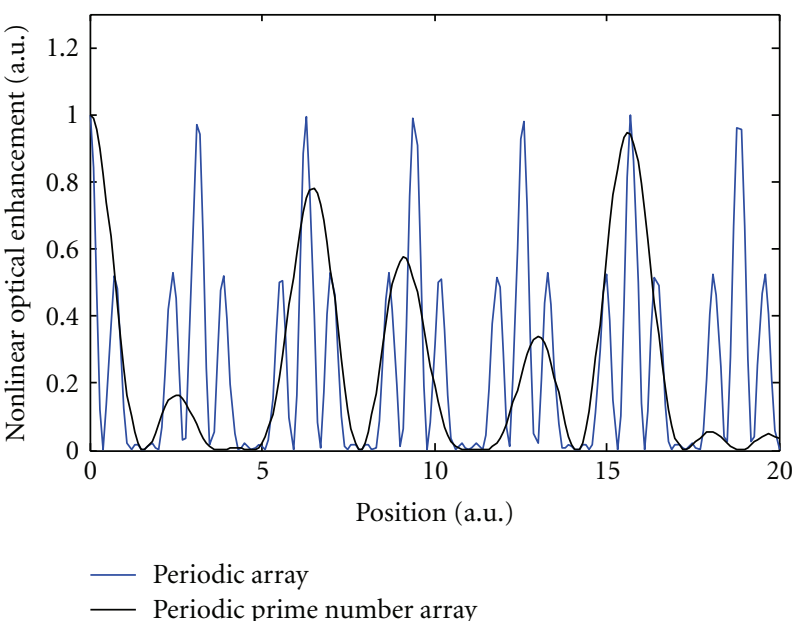

FIGURE 5: Third-order nonlinear optical response as a function of the distribution of the nanoclusters in a bilayer film.

in each film and the resulting rate relation between these two periodicities, $T_{A} / T_{B}$, is a pair number or a prime number.

Remarkable differences in the nonlinear optical response of aligned periodic arrays are showed in Figure 5. It is worth noting to mention that this contrast is absent when the comparison concerns to arrays with pairs or odds $T_{A} / T_{B}$ numbers. Nevertheless, pertaining to the studied prime number case, the distinctiveness is consequence from the irregular contributions from each particular region provided by the first nanocluster-containing film to the second one in a bilayer system of samples. Similar deductions have been achieved for plasmonic arrays of nanoparticles [16, 17]. Regarding the expanded distribution of interacting nanoclusters in multilayer arrays of thin films, it could be derived an increase in the participation of the nanoclusters by the modification of the volume fraction with respect to the area of the total interacting sample. Therefore, the opportunity to give rise to an enhancement of multiphotonic interactions seems to be possible. We consider that the major role about the involvement of the nanoclusters in a cascade nonlinear optical effect is to promote radiative recombination and excitations concerning to quantum confinement, with the benefits on the optical Kerr response by stronger accumulations of irradiance in the whole sample.

\section{Conclusion}

Within this work, we identified important differences in the nonlinear optical properties exhibited by monolayer and bilayer silver doped zinc oxide thin solid films prepared by an ultrasonic spray pyrolysis technique. A vectorial two-wave mixing experiment allows us to modulate the optical transmittance of a nonresonant $532 \mathrm{~nm}$ wavelength of excitation in the samples for evaluating the magnitude of the third order nonlinearities. We observed that a strong enhancement in the nonlinear optical response of zinc oxide can be obtained by silver doping and by prime number arrays in multiwave mixing interactions. Apparently, the thickness and the nanostructured morphology of the thin solid films 
originate a strong modification of the third order optical phenomena exhibited by zinc oxide thin films. Potential applications for developing all-optical devices can be contemplated.

\section{Acknowledgments}

The authors kindly acknowledge the financial support from Instituto Politécnico Nacional, COFAA-IPN, Centro de Investigación y de Estudios Avanzados del Instituto Politécnico Nacional, Instituto de Ciencia y Tecnología del DF through grants PIUTE10-129 and PIUTE-082, and Consejo Nacional de Ciencia y Tecnología. The authors are also thankful to the Central Microscopy facilities of the Centro de Nanociencias y MicroNanotecnologías del IPN.

\section{References}

[1] R. W. Boyd, Nonlinear Optics, Academic Press, San Diego, Calif, USA, 1992.

[2] M. B. de La Mora, J. A. del Río, R. Nava et al., “Anomalous patterned scattering spectra of one-dimensional porous silicon photonic crystals," Optics Express, vol. 18, no. 22, pp. 22808 22816, 2010.

[3] H. Rigneault, J.-M. Lourtiouz, C. Delalande, and A. Leven, Nanophotonics, ISTE Ltd, Newport Beach, Calif, USA, 2006.

[4] M. A. Reed, J. N. Randall, R. J. Aggarwal, R. J. Matyi, T. M. Moore, and A. E. Wetsel, "Observation of discrete electronic states in a zero-dimensional semiconductor nanostructure," Physical Review Letters, vol. 60, no. 6, pp. 535-537, 1988.

[5] H. C. Ramirez, R. Ramirez-Alarcon, M. Corona, K. GarayPalmett, and A. B. U'Ren, "Spontaneous parametric processes in modern optics," Optics \& Photonics News, vol. 22, pp. 36-41, 2011.

[6] B. Claflin, D. C. Look, and D. R. Norton, "Changes in electrical characteristics of $\mathrm{ZnO}$ thin films due to environmental factors," Journal of Electronic Materials, vol. 36, no. 4, pp. 442445, 2007.

[7] B. M. Monroy, G. Santana, A. Benami et al., "Photoluminescence of as-grown silicon nanocrystals embedded in silicon nitride: influence of atomic hydrogen abundance," Journal of Nanoscience and Nanotechnology, vol. 9, no. 5, pp. 2902-2909, 2009.

[8] C. Torres-Torres, L. Castañeda, and R. Torres-Martínez, "Evolution of the optical response in a nanostructured fluorine doped zinc oxide thin film," Semiconductor Science and Technology, vol. 27, no. 11, p. 115016, 2012.

[9] E. Fortunato, A. Gonçalves, A. Pimentel et al., "Zinc oxide, a multifunctional material: from material to device applications," Applied Physics A, vol. 96, no. 1, pp. 197-205, 2009.

[10] Ü. Özür, Y. I. Alivov, C. Liu et al., "A comprehensive review of ZnO materials and devices," Journal of Applied Physics, vol. 98, no. 4, Article ID 041301, pp. 1-103, 2005.

[11] K. Kyoungwon, C. D. Pulak, L. Deuk-Hee, K. Sangsig, and Y. L. Sang, "Effects of silver impurity on the structural, electrical and optical properties of $\mathrm{ZnO}$ nanowires," Nanoscale Research Letters, vol. 6, p. 552, 2011.

[12] L. Castañeda, M. Trejo-Valdez, and C. Torres-Torres, "Optical and photoconductiveproperties exhibited by silver doped zinc oxide thin films," Journal of Optics. In press.

[13] C. Torres-Torres, M. Trejo-Valdez, H. Sobral, P. SantiagoJacinto, and J. A. Reyes-Esqueda, "Stimulated emission and optical third-order nonlinearity in Li-doped $\mathrm{ZnO}$ nanorods," Journal of Physical Chemistry C, vol. 113, no. 31, pp. 1351513521, 2009.

[14] A. Smith and R. Rodriguez-Clemente, "Morphological differences in $\mathrm{ZnO}$ films deposited by the pyrosol technique: effect of HCl," Thin Solid Films, vol. 345, no. 2, pp. 192-196, 1999.

[15] L. Irimpan, V. P. N. Nampoori, and P. Radhakrishnan, "Spectral and nonlinear optical characteristics of $\mathrm{ZnO}$ nanocomposites," Science of Advanced Materials, vol. 2, no. 2, pp. 117$137,2010$.

[16] C. Torres-Torres, L. Tamayo-Rivera, R. Rangel-Rojo et al., "Ultrafast optical phase modulation with metallic nanoparticles in ion-implanted bilayer silica," Nanotechnol, vol. 22, no. 35, Article ID 355710, 2011.

[17] C. Forestiere, G. F. Walsh, G. Miano, and L. Dal Negro, "Nanoplasmonics of prime number arrays," Optics Express, vol. 17, no. 26, pp. 24288-24303, 2009. 

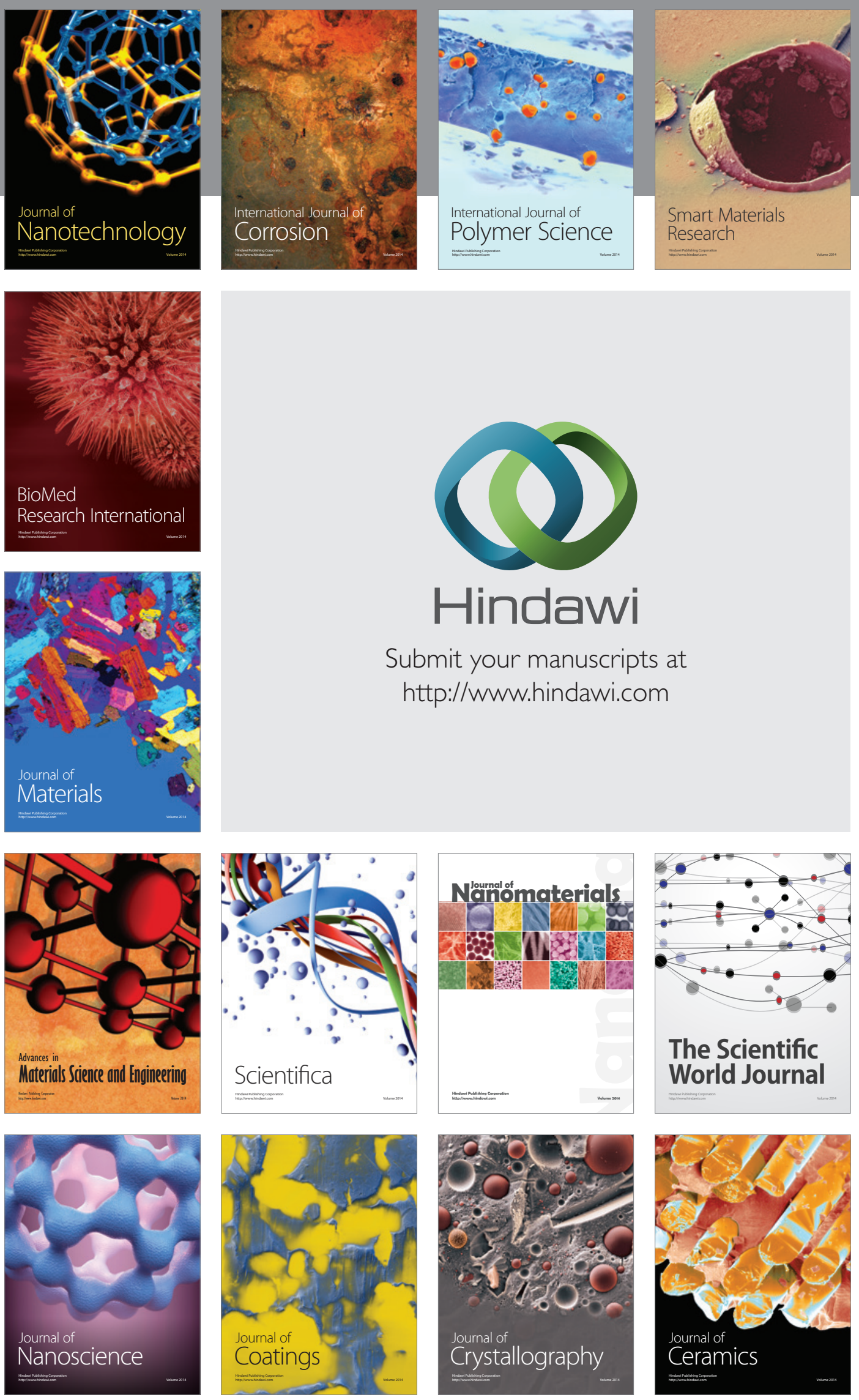

The Scientific World Journal

Submit your manuscripts at

http://www.hindawi.com

\section{World Journal}

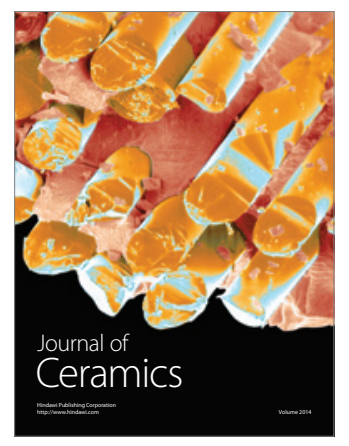

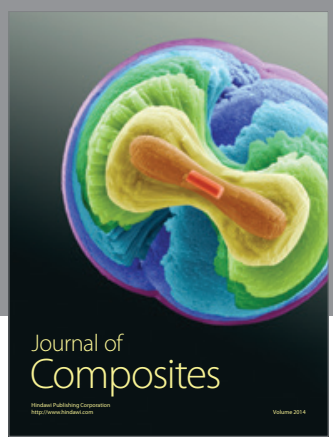
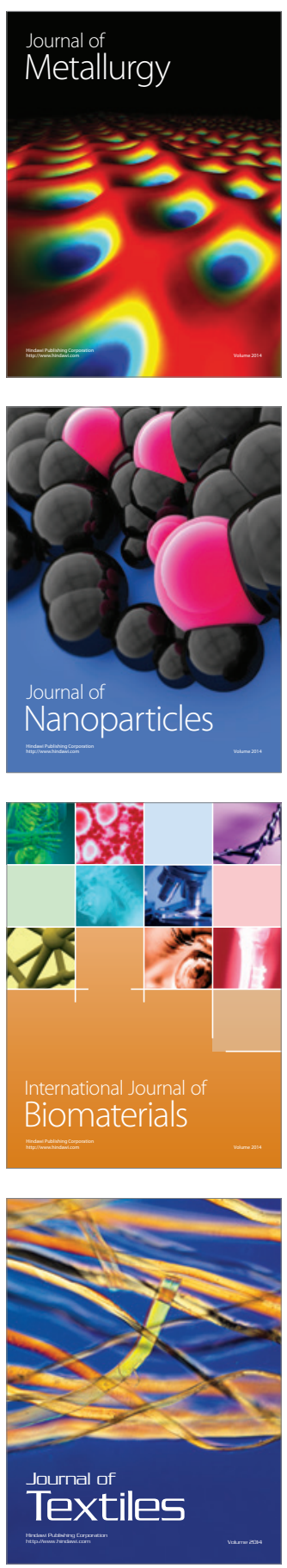